\title{
Mobility Support in Content Centric Networks
}

\author{
Do-hyung Kim \\ CS Dept. KAIST \\ Daejeon, South Korea \\ mr.dhkim@gmail.com \\ Hyun-soo Yoon \\ CS Dept. KAIST \\ Daejeon, South Korea \\ hyoon@cs.kaist.ac.kr
}

\author{
Jong-hwan Kim \\ ETRI \\ Daejeon, South Korea \\ shine1145@gmail.com
}

\author{
Yu-sung Kim \\ CE Dept. Sungkyunkwan Univ. \\ Suwon, South Korea \\ yskim525@gmail.com
}

\author{
Ikjun Yeom \\ CE Dept. Sungkyunkwan Univ. \\ Suwon, South Korea \\ ijyeom@gmail.com *
}

\begin{abstract}
Content-centric networking (CCN) is designed for efficient dissemination of information. Several architectures are proposed for CCN recently, but mobility issues are not considered sufficiently. We classify traffic types of CCN into real-time and non real-time. We examine mobility problems for each type, and suggest the possible hand-off schemes over CCN. Then, we analyze the delay performance in terms of simulation study. We believe that the proposed schemes can be merged as a part of the CCN easily, since they comply with the inherent nature and rules of the $\mathrm{CCN}$.
\end{abstract}

\section{Categories and Subject Descriptors}

[C.2.1 Computer Communication Networks]: Network Architecture and Design

\section{General Terms}

Design

\section{Keywords}

Information-centric networks, Mobility, Seamless Handoff

\section{INTRODUCTION}

Content-centric network(CCN) $[1,2]$ has emerged as a new communication paradigm in which a content is accessed by a name of the content instead of an IP address of the host having the content. This new networking concept is expected to utilize network bandwidth efficiently by separating contents from a specific location, and to decrease communication cost for the access to popular contents. The features of CCN introduce a lot of beneficial changes such as radical decrease of

*This work was supported by the National Research Foundation of Korea (NRF) grant funded by the Korea government (MEST) (No. 2012004958).

Permission to make digital or hard copies of all or part of this work for personal or classroom use is granted without fee provided that copies are not made or distributed for profit or commercial advantage and that copies bear this notice and the full citation on the first page. To copy otherwise, to republish, to post on servers or to redistribute to lists, requires prior specific permission and/or a fee.

ICN'12, August 17, 2012, Helsinki, Finland.

Copyright 2012 ACM 978-1-4503-1479-4/12/08 ...\$15.00. network congestion, fast dissemination of information, and secure access to contents.

CCN still has several aspects to be studied further for practical applications, and mobility is one of them. In CCN, contents are requested without a host identifier bounded by location information. Therefore, a mobile user does not need to update its location information during handoff and, can continue to request the content in a new location. Obviously, $\mathrm{CCN}$ is designed to support mobility of data requester fundamentally. However, seamless handoff for satisfying user's requirements is a different matter. Besides, if a data source moves, real-time services cannot be serviced without an additional strategy for updating the location information.

As of yet, there have not been enough researches for mobility support in CCN. In [3], a proxy-based mobility management scheme is proposed for CCN. This approach is designed to support user side (data requester) mobility with the help of a proxy server. In this scheme, control messages are delivered to the proxy server via IP address. In [4], an indirection approach is presented for supporting mobility of source nodes. This approach assumes that all the data sources are registered to a ISP's permanent server, and the top-level component of hierarchical data name in mobile sources points to this server. Then, all the interests heading for the mobile sources first arrive at this ISP's permanent server. This server maintains a set of binding information between persistent data names and temporary names which points to the current location. This server encapsulates an original interest with the temporary name and tunnels it toward the mobile source. This approach is a simple emulation of mobile IP [5] for $\mathrm{CCN}$, and the indirection point corresponds to a home agent. The limitations of the scheme are that the encapsulation of an original interest is not easy to be implemented in CCN because of the predefined interest format, and the handoff delay and system overhead are not considered. We compare this scheme with several other schemes including our proposed one.

In this paper, we look at mobility scenarios in $\mathrm{CCN}$ in Section 2, and suggest possible handoff schemes which operate in pure CCN in the Section 3 and Section 4. The proposed schemes deal with the mobility of data sources as well as data requesters and they are analyzed in terms of handoff latency and overhead. Particularly, we focus on the handoff scheme which minimizes handoff latency without packet loss for implementing seamless handoff in CCN in Section 5. In Section 7 , we conclude this paper. 


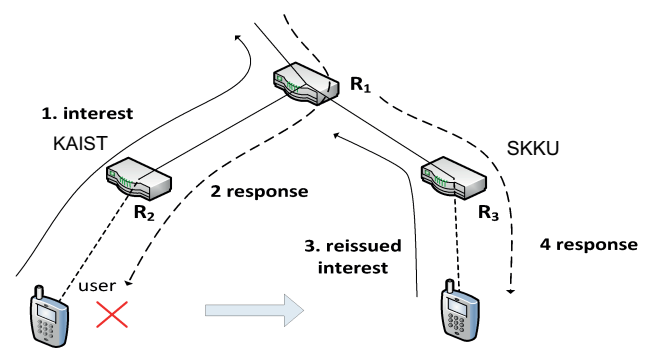

Figure 1: the mobility of a user

\section{BACKGROUND}

\subsection{Basic routing in $\mathrm{CCN}$}

In $\mathrm{CCN}$, communication is initiated by issuing a request messages (refers to interest). An interest is routed by a data name instead of a host identifier. Because the data name has a hierarchical structure like a file system pathname such as '/kaist.ac.kr/cnlab/ccnpaper', each CCN router can forward an interest in a hop-by-hop manner. If a $\mathrm{CCN}$ router has a copy of the requested data in its local cache, it sends the requested data (refers to response) immediately. Otherwise, it forwards the interest to the next CCN router toward the data source by longest prefix match based on Forwarding Information Base (FIB). Whenever the interest is relayed, a $\mathrm{CCN}$ router maintains the interest in Pending Interest Table (PIT) for routing the response along the reverse directional path.

\subsection{Caching in the router}

In-network caching is one of the core functionality in CCN for efficient distribution of contents. When a CCN router delivers data packets, the CCN router also stores the data in its local cache in order to serve later accesses to that data. The data resides in the cache until the valid period (TTL) of the data expires, or the buffer is overflowed. TTL of each data is set by the application. For efficient use of the network, an application should assign TTL carefully.

\subsection{Mobility problems in CCN}

Considering the impact of handoff delay to the services, we classify contents into 'stored contents' and 'real-time contents' based on the time sensitiveness. 'Stored contents' are the contents that are relatively free from a delay constraint. Web pages, e-mails, and files are typical examples of stored contents. These contents are usually served by well-known persistent content providers, and their locations are relatively stable. Hence, the mobility problem for the 'stored contents' is limited to the scope of user side mobility. Fortunately, this problem can be solved by simply retransmitting interests. In Fig.1, a mobile user moves from the access point router $R_{2}$ to the access point $R_{3}$ after issuing an interest. While a response is delivered to $R_{2}$, the response is stored in caches of intermediate routers for the future request. If the mobile user moves within a subnet, an intermediate router can immediately respond to the retransmitted interest. If the user moves to another network, the data can be served by the data source with a little longer delay.

On the other hand, 'real-time contents' should be delivered in a very short limited time. Internet telephony, messaging and online gaming are typical examples of real-time contents. These contents are created and served by individual mobile devices unlike 'stored contents'. Therefore, the mobility of data sources should be considered as well. If a data source changes its location, a hierarchical path name(routable prefix) may also change. Otherwise, FIB entries cannot be aggregated and the size of FIB becomes explosive. If a data source has a new name for the content, the user cannot request the content until it is informed of the new content name by the data source. Hence, the communication is blocked for more than a round trip time at least. The typical round trip time on the current Internet is $200 \mathrm{~ms}$ and the acceptable delay in voip application is less than $150 \mathrm{~ms}$. Although the round trip time in CCN is expected to become smaller than that in IP based network, this delay cannot be negligible.

When the two communication peers perform handoff at the same time, what is worse is that they cannot exchange their new routable prefixes directly. So, in the example of voice over CCN, a caller and a callee first inform a registration server of their new routable prefixes after the handoff. Then, the caller obtain the new routable name of the callee via the registration server and can send an interest to the callee. This process incurs huge handoff delay and disrupts the Internet telephony service obviously. Here, we note that from now on we refer a service initiator as a caller and the communication peer as a callee for the convenience of explanation, even though our schemes are not restricted to the Internet telephony service.

\subsection{Rendezvous point for mobile users}

In order to initiate a real-time service, a caller needs to know the target content name which includes a hierarchical path name. As the hierarchical path name could be changed by the mobility of a callee, a caller should achieve the content name from a well-known server that keeps track of the location information of all service clients. We refer this location management server as rendezvous point. Generally a rendezvous point provides only a naming service which provides the location information of a communication peer. In [4], however, a rendezvous point intervenes in relay of all the data packets (interest and response) in order to handle source mobility. We refer this type of rendezvous point as indirection point for the convenience of explanation. And we look at the mobility schemes using the rendezvous point and the Indirection point and compare their performance.

\subsection{Sender-driven control message}

In $\mathrm{CCN}$, communication is driven by a receiving end. However, if a caller changes its routable prefix, no entity in a network cannot send an interest first. Hence, sender-driven message is required for supporting server-side mobility. To implement sender-driven messaging, we borrow the scheme used in an e-mail system in CCN [6]. For an e-mail service from SKKU to KAIST address 'dhkim@kaist.ac.kr', the email sender(ijyeom) first issues an interest with the name, 'kaist.ac.kr/email_rcpt/dhkim/skku.edu|ijyeom|\#1234'. This name includes additional parameters which are necessary for starting a receiver-driven communication. Similarly, information required for supporting mobility is delivered in the same way like 'kaist.ac.kr/handoff/(parameters)'. Then, the corresponding daemon can translate this name in the predefined format. 


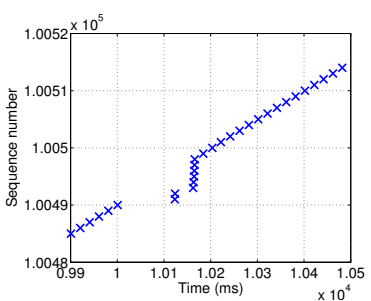

(a) Caller

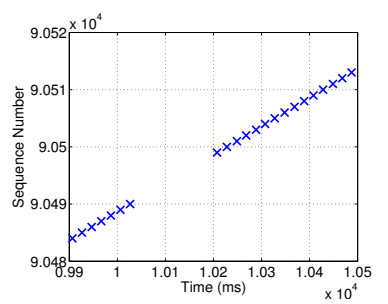

(b) Callee
Figure 2: When only a caller moves

\subsection{Interest retransmission for reliable hand- off}

For reliable handoff, it is necessary to recover the lost data chunks during the handoff. In that case, the handoff latency is largely dependent on the timeout value of the loss detection timer $\left(L_{d}\right)$ as the recovery process is initiated by the expiration of loss detection timer. The small value of $L_{d}$ enables a fast handoff. However, controlling $L_{d}$ is not simple because it is relevant to the lifetime of PIT entry $\left(L_{p}\right)$. If $L_{d}$ is smaller than $L_{p}$, the retransmitted interest for recovery cannot be forwarded to the data source because intermediate routers which already have a PIT entry for the same content aggregate that retransmitted interest. Hence, lower bound of $L_{d}$ is determined by $L_{p}$. The default value of $L_{p}$ is known to be 4 second in the current implementation of $\mathrm{CCNx}$, but it can be controlled by 'interestlifetime' field in the interest packet. For the fast reliable handoff, therefore, $L_{p}$ should be set as the slightly larger value than the RTT, which is for routing the response.

Another approach for fast reliable handoff is using a explicit marking bit which indicates whether an interest is a retransmitted one or not. If the bit is set, then the CCN routers forward it to the data source even though they already have the PIT entry of the same content. This simple approach works well for the unicast type of services such as voice over CCN. However, in the multicast type of services where many callers receive the contents from a callee at the same time, too many retransmitted interests can cause implosion problem, which is the identical to the NACK implosion problem reported in [7]. The essential problem of the NACK implosion is simultaneous arrival of too many duplicated packets. In order to prevent implosion problem, CCN routers maintain the time when the retransmitted interest is relayed in PIT and aggregate the duplicated retransmitted interests during the interval $L_{r}$. Then, the number of duplicate retransmitted interests arrived at the source node shrinks down to $\frac{1}{L_{r} R}$, where $R$ is the arrival rate of the retransmitted interests. The proposed scheme using the explicit marking bit can decouple $L_{d}$ value from $L_{p}$ so that a fast recovery can be implemented regardless of $L_{p}$.

\section{MOBILITY WITH RENDEZVOUS POINT}

\subsection{User side mobility}

User side mobility for the stored contents in CCN is supported by simple retransmission of interests as we mentioned above. Fig. 2(a) shows the sequence number of the contents that a caller receives from a callee after the caller's handoff.

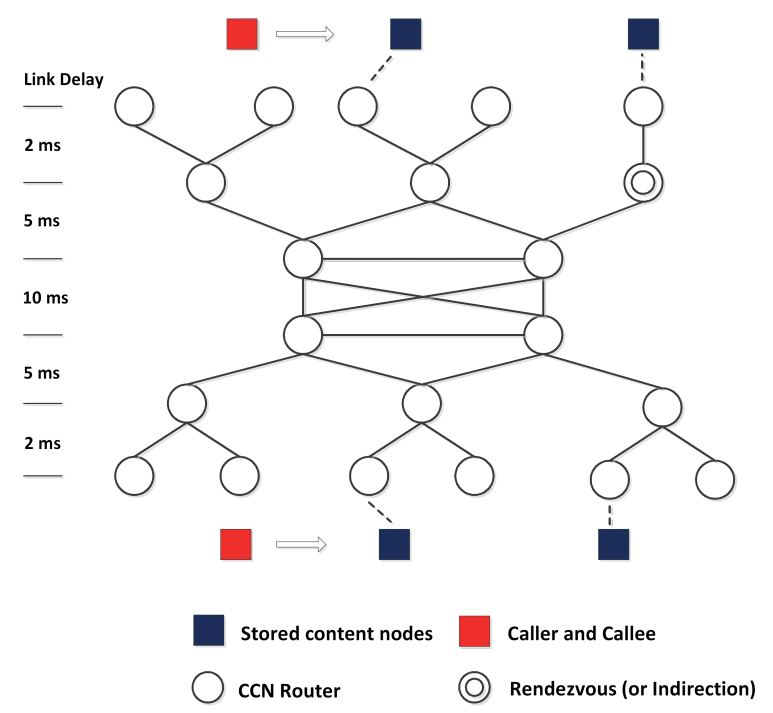

Figure 3: topology
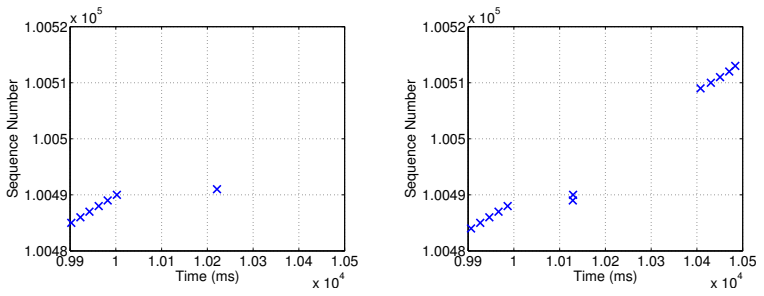

Figure 4: simultaneous Figure 5: query to Renhandoff dezvous

The simulation topology considered in this paper is shown in Fig. 3. L2 Layer handoff delay is $100 \mathrm{~ms}$ and handoff starts at 10 sec. If a loss detection timer expires, the caller retransmits interests for the lost data chunks at the new location. The first two responses after the handoff are received from the in-network cache. And rest of the recovered responses are received from the callee. In this simulation, $L_{d}$ and $L_{p}$ are statically set as $100 \mathrm{~ms}$.

\subsection{Direct exchange for location update}

Form the callee's point of view, the caller's handoff implies the mobility of data source. Therefore, the callee should be informed of a new hierarchical path name of the caller in order to continue an ongoing service. Fig. 2(b) shows the result when the caller notifies the new hierarchical path name directly to the callee. It is shown that the callee can continue receiving responses after the handoff period. Unlike the caller side, the lost data chunk cannot be recovered by simple retransmission of interests unless the application regenerates the interests with the new hierarchical path name. Direct notification method seems quite simple and effective but it causes a serious problem when both communication peers perform handoff simultaneously.

Fig. 4 shows the result when simultaneous handoffs perform. Under direct notification method, both communication peers send their new hierarchical path name to the previous location of their peer after the handoff. Resultingly, both communication peers cannot reach each other and the 
service cannot continue. Here we note that the sequence number appeared after the handoff means that a single data chunk which lost the way has been in the network cache and finally is delivered to the peer in response to the retransmitted interest.

\subsection{Query to the Rendezvous for location up- date}

If a caller initiates a service to the mobile callee, the caller first sends a query to a rendezvous server in order to obtain the callee's location information. In order that the rendezvous server keeps track of the location of all clients, a new hierarchical path name of the client is informed to the rendezvous server whenever a handoff event occurs. Therefore, using the rendezvous server the routable prefix of the peer can be achieved even after simultaneous handoffs. Under this scheme, the query to the rendezvous is triggered if a client has not received any response during a predefined time interval $\left(L_{h}\right)$. In the simulation, we set $L_{h}$ as a larger value than the handoff delay (L2 handoff delay + RTT). Fig. 5 shows the result when the rendezvous server is utilized. Due to the subtle difference in both peers' handoff timing and RTT to the Rendezvous server, the Rendezvous server can deliver outdated information to the client. In that case, the client should retry issuing interests with the wrong hierarchical path name, and then query again after $L_{h}$. Therefore, the maximum possible handoff latency in this scheme is $2 L_{h}+2 \mathrm{RTT}$, which is close to $400 \mathrm{~ms}$. Besides, like in the previous approach, regeneration of an interest with the new hierarchical path name is required to recover the lost data chunk instead of simple retransmission of an existing interest.

\section{MOBILITY WITH INDIRECTION POINT}

It is shown that the handoff solution using the rendezvous server induces relatively large handoff latency. In order to minimize the handoff latency, the indirection server can be considered. In the environment with an indirection server, a caller issues an interest with the name '(indirection point name $) /($ callee $) /($ content Id)' in order to initiate a request. If the indirection server receives the interest, it buffers that interest and issues another new interest toward the callee with the name '(callee's routable name)/(content id)'. After receiving the content as a response from the callee, the indirection server generates a corresponding response to the buffered interest and send them to the caller. When a caller's handoff is imminent, the caller notifies a handoff event to the indirection server. Since then, if the indirection server receives an interest heading for the caller, it just buffers that interest and postpones making a corresponding interest toward the caller. After the new hierarchical path name is informed by the caller, the indirection server resumes to generate corresponding interests toward the caller and continues the normal service as usual.

Fig. 6 shows the result of the Indirection scheme when handoffs are performed simultaneously at both communication peer. As the callee locates closer to the indirection server than the caller, the indirection server can achieve the callee's contents first after handoff events. Therefore, the caller's retransmitted interests are readily responded by the Indirection server. However, in order to serve the callee's retransmitted interests, the RTT between the indirection server and the caller is additionally required after finishing

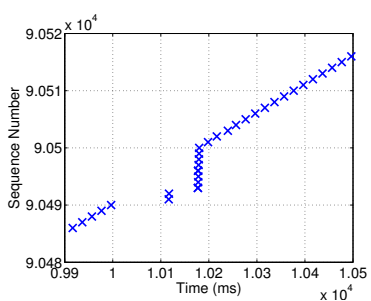

(a) Caller

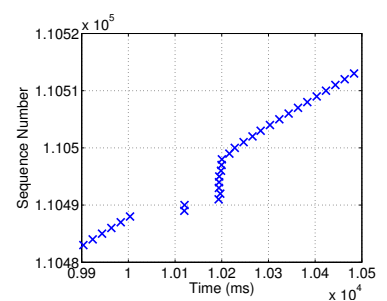

(b) Callee
Figure 6: Indirection point

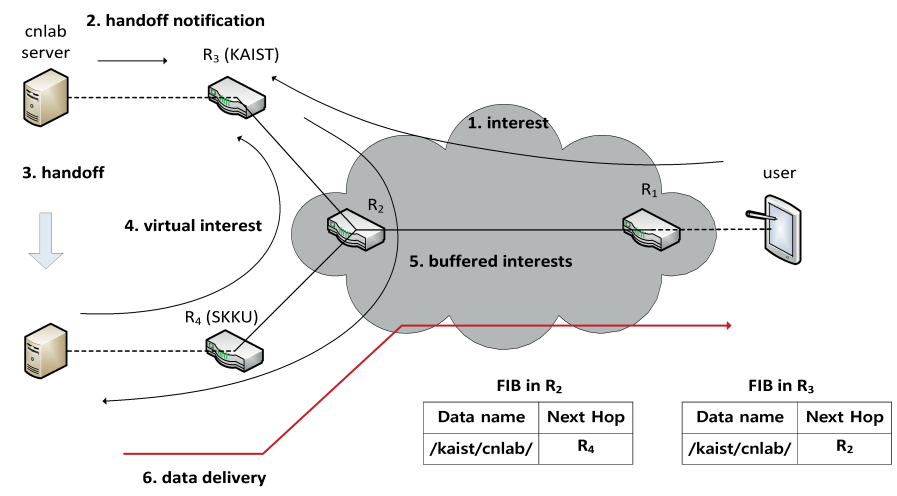

Figure 7: Interest forwarding scheme

the caller's handoff. This is because the caller has smaller handoff latency compared with the callee.

The maximum handoff latency is about $226 \mathrm{~ms}$ in this scheme. This shorter handoff delay and the recovered data chunks indicates that the indirection server can support handoff more efficiently than the rendezvous server. However the problem of the indirection point scheme is longer average RTT. As all the data should be exchanged via the indirection server, the maximum possible RTT is almost two times of the RTT in previous schemes, which is $96.83 \mathrm{~ms}$. Besides, the indirection point becomes a single point of failure as all the traffic are concentrated on it.

\section{INTEREST FORWARDING}

The longer average RTT in the Indirection point scheme is too much cost for improving handoff performance. Hence, we suggest a new CCN layer approach, referred to 'Interest forwarding'. In previous schemes, a new hierarchical name is necessary to continue a service in the new location. This is because intermediate CCN routers keep FIB as an hierarchically aggregated form instead of all the independent entry for each content. Therefore, the interest cannot be reached to the handoffed client without changing the hierarchical path name. However, we have seen that changing a content name requires time demanding steps such as DHCP process and registration to the rendezvous server, so that it makes seamless handoff difficult. So, we introduce a new handoff support scheme which does not require a new hierarchical name even after changing networks.

'Interest forwarding' scheme starts from the handoff notification process. In Fig.7, a mobile client notifies a handoff event to $R_{3}$ when handoff is imminent. Then $R_{3}$ starts to 


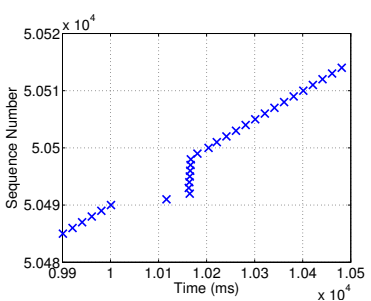

(a) Caller

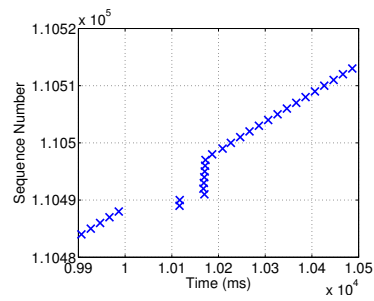

(b) Callee
Figure 8: Interest forwarding

buffer the incoming interests heading for the mobile client. After the mobile client changes its access router from $R_{3}$ to $R_{4}$, it issues a virtual interest with the name " $/$ kaist.ac. $\mathrm{kr} / \mathrm{c}$ $n l a b /$. A virtual interest forwards to $R_{3}$ by the longest prefix matching based on FIB. When the intermediate routers in the path between $R_{3}$ and $R_{4}$ receive the virtual interest, they update their FIB in order to make packets for cnlab destined to $R_{4}$. When the virtual interest arrives at previous access router $R_{3}$, the buffered interests heading for $c n l a b$ start to forward to cnlab without a new hierarchical path name of cnlab.

However, the intermediate router $R_{2}$ may discard the buffered interests in order to prevent routing loop, as it already has a PIT entry for the same content name. Hence, a bit for indicating retransmission, which is mentioned in Section 2.6, is set for the buffered interests in order to prevent them from being aggregated. When responses are delivered along the reverse path, they forward to the user directly because PIT in $R_{2}$ leads them to the user. The subsequent interests are forwarded from the user to $R_{4}$ directly because the routing path to the mobile source is successfully updated.

A FIB entry that is created by a virtual interest has a lifetime in order to prevent radical increment of FIB. The lifetime is specified in 'interestLifetime' field of an virtual interest and it can be extended by periodical transmission of the virtual interest. After ongoing communication finishes, the mobile data source performs naming process. Then, it does not issue the virtual interest and the entry is deleted in FIB automatically.

Fig. 8 shows the result of interest forwarding when simultaneous handoffs occur. In Fig. 8(a), the first data chunk after the handoff is delivered in response to the buffered interest at the previous access router. The rest data chunks to be recovered are responses to the retransmitted interests at the caller. This result implies that without changing the data name of callee, the source mobility can be supported. The handoff delay until getting the data chunks continuously is $178 \mathrm{~ms}$, which is the minimum value compared with the handoff latency of previous approaches. Besides the average RTT value is preserved as $54.43 \mathrm{~ms}$.

'Interest forwarding' scheme requires the modification of CCN routers because CCN routers should buffer incoming interests toward a handoff client and process a virtual interest. But it has following advantages: 1) It minimizes the path update overhead because a new entry is temporarily added only to the routers on the path between the access routers that are relevant to the handoff; 2) As a new routable prefix is not required during the handoff process, handoff latency is minimized; 3) Triangular routing is prevented automatically.

\section{MISCELLANEOUS}

\subsection{Simultaneous transmission using multiple interface}

If a client performs handoff between different kinds of networks such as $3 \mathrm{G}$ to wi-fi, seamless handoff can be implemented by simultaneous transmission of copied interests using multiple interfaces. As CCN allows multiple connections via all the network interfaces, a user can receive the same response from different interface cards in handoff zone, which realizes soft handoff [8] automatically.

\subsection{Lifetime of the realtime content}

Even though future requests for the real-time contents are hardly expected, they are cached in the network in order to enable fast recovery of lost data via retransmission. But we should be careful for determining the lifetime of the copied real-time contents as these copies are a waste of resources but for the loss recovery. Here, we suggest that the lifetime of realtime contents should be slightly larger than handoff delay (L2 handoff delay $+\mathrm{RTT} / 2$ ) in order to support the fast recovery of packet loss due to the handoff.

The lifetime is set as the following way. When a handoff is imminent, the handoff client sets a specialized bit in the sending interests. And the user also specifies the TTL of copies in the 'interestlifetime' field. When a data source receives the interest, it sets the TTL of the real-time content as the value in the 'interestlifetime' field in order to maintain the copies during handoff period. Here, we note that the 'interestlifetime' field is originally used to indicate the time remaining before the interest times out. Therefore, the lifetime of an interest in PIT table becomes a little longer. But we expect that the effect of it is insignificant.

\section{CONCLUSION}

Basically, CCN is designed to support the mobility of data requesters. But lots of application also requires the mobility of data sources and handoff latency should be minimized for real-time contents. In this paper, we present several handoff scenarios to support real-time services in CCN and compare their performance. Via the simulation study, we have shown that the 'interest forwarding' outperforms other possible schemes.

\section{REFERENCES}

[1] V.Jacobson et al., "Netwroking named content," in $A C M$ CoNEXT, 2009

[2] D.Smetters and V.Jacobson, "Securing network content," PARC, Tech. Rep., 2009.

[3] J.Lee et al., "Proxy-based mobility management scheme in mobile content centric networking (CCN) environments," in ICCE, 2011.

[4] Hermans et al., "Mobile sources in an information-centric network with hierarchical names: An indirection approach," in $S N C N W, 2011$.

[5] D.Johnson et al., "Mobility support in ipv6 (RFC 3775)," IETF, Tech. Rep., Jun. 2004.

[6] "Named data networking project," 2011, [Online]. Available: http://www.named-data.net/.

[7] L. Lehman et al., "Active reliable multicast," in INFOCOM, 1998.

[8] I. Akyildiz, "Mobility management in current and future communication networks," IEEE Network, vol. 12, pp. 39 49, 1998. 